\title{
Correlations between Thermal Conductivity and Inelastic Deformation of Aluminum Based Composites Containing VGCF-CNT Network*
}

\author{
Kohei FUKUCHI**, Katsuhiko SASAKI**, Terumitsu IMANISHI***, \\ Kazuaki KATAGIRI***,**** and Atsushi KAKITSUJI***** \\ **Division of Human Mechanical Systems and Design, Hokkaido University \\ Kita 13, Nishi 8, Kita-ku, Sapporo, Hokkaido, 060-8628, Japan. \\ E-mail: k_fukuchi@mech-me.eng.hokudai.ac.jp \\ ***Sumitomo Precision Products Co., Ltd. \\ 1-10, Fuso-cho, Amagasaki, Hyogo, 660-0891, Japan. \\ ****Technology Research Association for Single Wall Carbon Nanotubes \\ AIST Tsukuba Central 5, 1-1-1 Higashi Tsukuba, Ibaraki, 305-8565, Japan. \\ *****Technology Research Institute of Osaka Prefecture \\ 2-7-1, Ayumino, Izumi, Osaka, 594-1157, Japan
}

\begin{abstract}
This paper evaluates the reliability of an aluminum based composite including vapor growth carbon fibers (VGCF) and carbon nanotubes (CNT). The composite is fabricated using spark plasma sintering and has high thermal conductivity. For the reliability evaluation, the correlation between the inelastic deformation and thermal conductivity of the composite is discussed both with experiments and simulation conducted by the finite element method (FEM). Specimens made from the composite are first subjected to tensile loading until inelastic strain occurs. After the tensile loading, the thermal conductivities of the specimens were measured to establish the differences between the thermal conductivity before and after the tensile loading. The FEM analyses are also conducted to evaluate the reliability of the composites. It was found that the thermal conductivity changed due to the inelastic deformation of the composites and that a FEM analysis considering the damage due to the deformation qualitatively estimates the differences in the thermal conductivity before and after the tensile loading.
\end{abstract}

Key words: Aluminum Matrix Composite, Carbon Nanotube, Vapor Growth Carbon Fiber, High Thermal Conductivity, Finite Element Method, Inelastic Deformation

\section{Introduction}

Improvements in energy efficiency are a significant factor to enable reductions in carbon dioxide generation. In heat exchange systems such as radiators and fins, an effective way to improve the energy efficiency is to use materials which have higher thermal conductivities. Carbon nanotube (CNT) [1] is one of effective materials to increase the thermal conductivity of materials used in radiators and fins because CNT has characteristics such as high thermal conductivity, high electrical conductivity, and high strength.

The thermal conductivity and mechanical characteristics of composites containing CNT have been widely reported, and many kinds of polymer based composites have been developed. Stephan et al. [2] studied the mechanical properties of thin films of single walled carbon nanotubes (SWCNT)/poly methyl methacrylate (PMMA) composites using Raman 
spectroscopy and scanning electron microscopy (SEM). Hone et al. produced dense and thick films of aligned SWCNT with controlled alignment of the CNT in a strong magnetic field and measured the thermal conductivities. Hone et al. also made bulk samples of SWCNT and discussed the thermal conductivity both microscopically and macroscopically $[3,4]$. Biercuk et al. [5] estimated the thermal conductive properties and Vickers hardness of SWCNT/epoxy resin composites. Patton et al. [6] obtained values for heat decomposition, ablation, thermal conductivity, and mechanical properties of vapor growth carbon fiber (VGCF)/phenolic resin composites. Chen et al. [7] obtained the thermal conductive properties of carbon/carbon composite with multi walled carbon nanotubes (MWCNT). Nam et al. [8] fabricated a poly butylene succinate (PBS)/coir fiber biodegradable composite and determined the tensile and flexural properties, thermal stability, and thermal expansion characteristics.

Recently, many kinds of metal matrix composites (MMC) containing CNT were studied. $\mathrm{Xu}$ et al. [9] described the relationship between the thermal contact conductance of $\mathrm{Cu} / \mathrm{CNT} / \mathrm{Si}$ and contact stress, experimentally. Wei et al. [10] fabricated MWCNT/ $/ \mathrm{Al}_{2} \mathrm{O}_{3}$ composites using a hot pressing method and determined the toughness and bending strength. Tokunaga et al. [11] fabricated CNT/Al composites using high pressure torsion to decrease the content of aluminum-carbide which causes decreases in the strength of the composites. Ogawa et al. [12] studied the thermal conductivity of VGCF/Al-Si composites, and Xu et al. [13] reported the Vickers hardness of VGCF/Al composites. Ueno et al. [14] determined the thermal conductivities of Al based composites containing different types of CNT such as SWCNT, MWCNT, and others.

Theoretical determinations of the thermal conductivity of composites have also been conducted: Singh et al. [15] simulated thermal conductivities of polymer based composites containing CNT using element-free Galerkin methods. Nan et al. [16-17] proposed a numerical method to estimate the mean thermal conductivities of particle dispersion composites and applied the method to composites containing CNT considering interfacial thermal resistance.

The relationship between material properties of composites and a microstructure is studied. Baney et al.[18] simulated a stress-strain relation of silicon-carbide (SiC)/alumina $\left(\mathrm{Al}_{2} \mathrm{O}_{3}\right)$ composite considering debonding occurred in the composite using micromechanical theory. Chang et al.[19] estimated an effect of $\mathrm{TiO}_{2}$ nanoperticle on microhardness of $\mathrm{Sn} 3.5 \mathrm{Ag} 0.7 \mathrm{Cu}$ solder considering microstructure.

The authors have fabricated Al matrix composites containing CNT and VGCF using spark plasma sintering (SPS), and showed that the composites had high thermal conductivities and further suggested that the high thermal conductivities arise from VGCF-CNT networks in the composite [20-22].

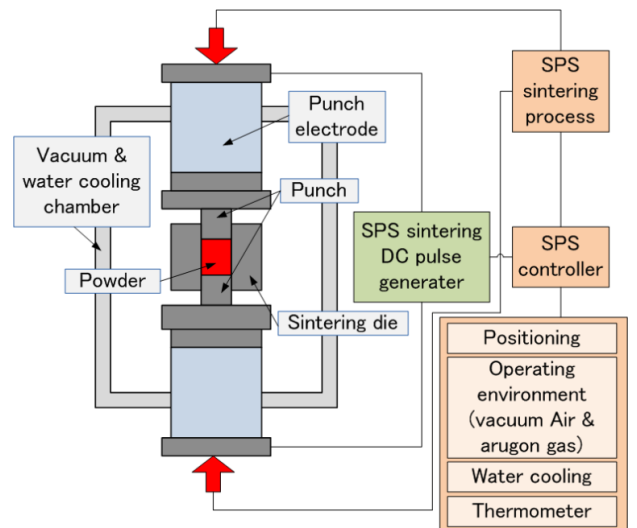

Fig. 1 Schematic outline of spark plasma sintering (SPS).

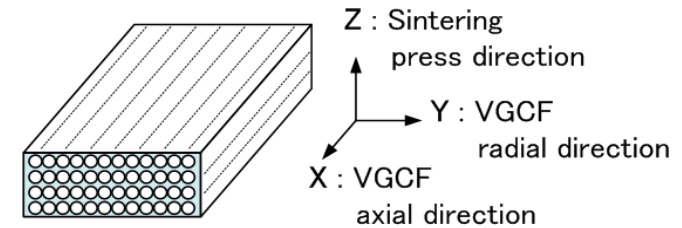

Fig. 2 Coordinate system for the composites. 


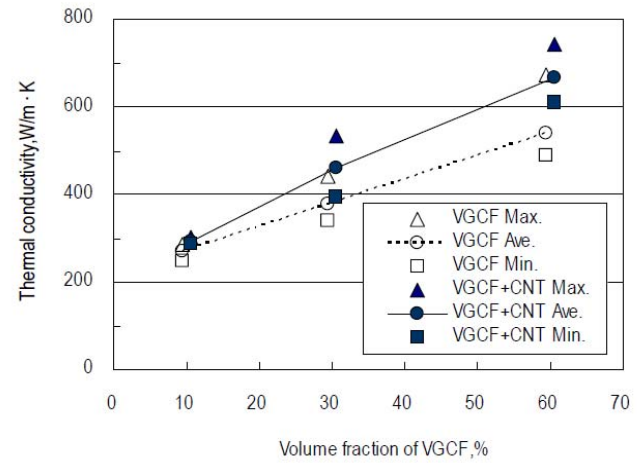

Fig. 3 Relationship between thermal conductivity and volume fraction of VGCF confirming an effect of CNT dispersion[21].

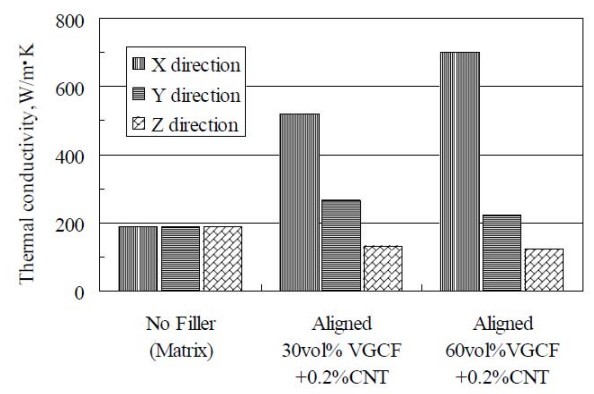

Fig. 4 Thermal anisotropy of the composites[21].

To apply the fabricated composites to real use conditions, it is important to establish correlations between inelastic deformation and thermal conductivity. In this study, the effect of inelastic deformation on the thermal conductivity of composites containing VGCF-CNT networks is investigated by experiments and simulation. First, the thermal conductivities of the composites are measured before and after inelastic deformation of the composites and the correlation between the inelastic deformation and thermal conductivity is determined. To understand the mechanism of the correlation between inelastic deformation and thermal conductivity, simulations with the finite element method (FEM) are conducted considering the damage occurring due to the inelastic deformation.

\section{Fabrication of VGCF/Al composites containing CNT [20], [21]}

\subsection{Fabrication methods}

The VGCF/Al composites here were fabricated by powder metallurgy using a spark plasma sintering (SPS) apparatus, a schematic outline of the SPS is shown in Fig. 1. SPS method can fabricate $\mathrm{VGCF} / \mathrm{Al}$ composite without aluminum-carbide due to the temperature of the sintering being relatively low $(833 \mathrm{~K})$ and the length of the sintering period short ( 90 minutes)[20]. The VGCF used in this work has a thermal conductivity of $2000 \mathrm{~W} / \mathrm{mK}$ and a tensile strength of $7 \mathrm{GPa}$. The composites are fabricated with aligned VGCF: the composites are manufactured by interbedding the aligned VGCF containing $\mathrm{CNT}$ and matrix powder. The mean sizes of the matrix powder particles of pure Al and $\mathrm{Al} / 12 \mathrm{Si}$ are $35 \mu \mathrm{m}$. Figure 2 shows the coordinate system for the composites: the $\mathrm{X}$ direction is the axial direction of the VGCF, $\mathrm{Y}$ is the radial direction of the $\mathrm{VGCF}$, and $\mathrm{Z}$ is the direction to the sintering press, respectively.

\subsection{Measurements of thermal conductivity}

The thermal conductivities are measured by laser-flash equipment (ULVAC, TC-7000). The density is measured by the Archimedean method to obtain the thermal conductivity.

Figure 3 showed the relationship between the volume fraction of the VGCF and the thermal conductivity in the axial direction of the VGCF[21]. In Fig. 3, the open circles showed the thermal conductivity of composites without CNT, and the solid circles were the thermal conductivity of composites containing $0.2 \%$ of CNT. In the case of $10 \%$ of VGCF, the thermal conductivity was $292 \mathrm{~W} / \mathrm{mK}$ with CNT and $271 \mathrm{~W} / \mathrm{mK}$ without CNT, giving the composites containing CNT having an $8 \%$ higher thermal conductivity than the composite without CNT. In the case of $60 \%$ of VGCF, the thermal conductivity was $666 \mathrm{~W} / \mathrm{mK}$ with $\mathrm{CNT}$ and $538 \mathrm{~W} / \mathrm{mK}$ without $\mathrm{CNT}$, resulting in the composites containing CNT having a 24\% higher thermal conductivity than composites without CNT. This showed that small amounts of CNT addition were effective to improve the thermal conductivity of the composites. 


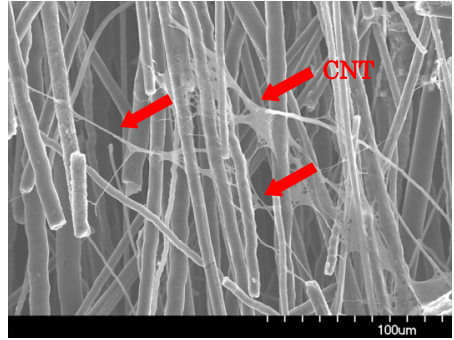

(a) SEM image[21]

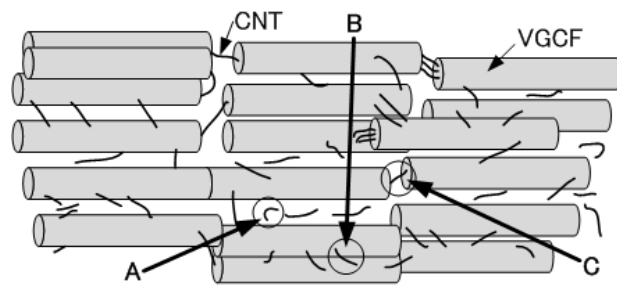

(b) schematic figure

Fig. 5 Schematic figure of VGCF-CNT network.

Figure 4 showed the thermal conductivities in the $\mathrm{X}, \mathrm{Y}$, and $\mathrm{Z}$ directions of the composites[21]. The composites displayed thermal anisotropy though the matrix does not have thermal anisotropy, i.e. the thermal conductivity in the $\mathrm{X}$ direction, the axial direction of the VGCF, was much larger than those of the $\mathrm{Y}$ and $\mathrm{Z}$ directions, which were along the radial directions of the VGCF. The thermal anisotropy of the composites was caused by the thermal anisotropy of the VGCF. There were also differences in thermal conductivity between the $\mathrm{Y}$ and $\mathrm{Z}$ directions: the thermal conductivity in the $\mathrm{Z}$ direction was smaller than that in the $\mathrm{Y}$ direction. The thermal conductivity of the $\mathrm{Z}$ direction was smaller than even that of the matrix. This is because the $\mathrm{Z}$ direction was the buildup direction of VGCF-CNT filler and the matrix layer exists between the filler parts as shown in Fig. 2.

\subsection{VGCF-CNT networks}

To understand the mechanism of the increases in the thermal conductivity of the composites containing both VGCF and CNT, SEM observation of the VGCF filler dispersed CNT was conducted. Figure 5(a) showed SEM micrograph of the VGCF filler sheet with dispersed CNT. The VGCF and CNT were blended and a VGCF-CNT network was clearly observed[21]. Lestriez et al. [23] and Wu et al. [24] studied networks between carbon fibers and CNT to improve the electric conductivity of electrodes for lithium batteries and showed that the network formed by the carbon fibers and CNT was affected in improving the electric conductivity. This suggested that the VGCF-CNT network may be a reason for the increase in the thermal conductivity of the composites. Figure 5(b) schematically suggested the VGCF filler with dispersed CNT. There may be three dispersed conditions of CNT: agglomerated CNTs (A in Fig. 5(b)) which would affect a decrease in the thermal conductivity negatively, cross-linked CNTs between the radial direction of VGCFs (B in Fig. 5(b)) which would not effect on the thermal conductivity, and cross-linked CNTs between the axial direction of VGCFs (C in Fig. 5(b)) which would affect an increase in the thermal conductivity positively. It was possible that inelastic deformation of the composites affected microstructures such as the agglomerated CNTs and VGCF-CNT networks and the thermal conductivities of the composites. Therefore, to make it possible to apply the composites to real use conditions the effect of the inelastic deformation on the thermal conductivity of the composites should be clarified.

\section{Effect of inelastic deformation on thermal conductivity}

\subsection{Experimental methods}

The effect of inelastic deformation on the thermal conductivity of the composites was first evaluated by experiments using tensile specimens as shown in Fig. 6.

A composite including a $30 \%$ volume fraction of VGCF was used in the experiments. At least seven specimens were prepared for the tensile tests. The specimens also included a $0.2 \%$ volume fraction of CNT, and Fig. 6 shows the geometry of the test specimens used here. The specimens for the tensile test have a gauge length of $25 \mathrm{~mm}$, a width of $25 \mathrm{~mm}$, and a thickness of $3 \mathrm{~mm}$, respectively. After the tensile tests, the thermal conductivities of the specimens were measured. 


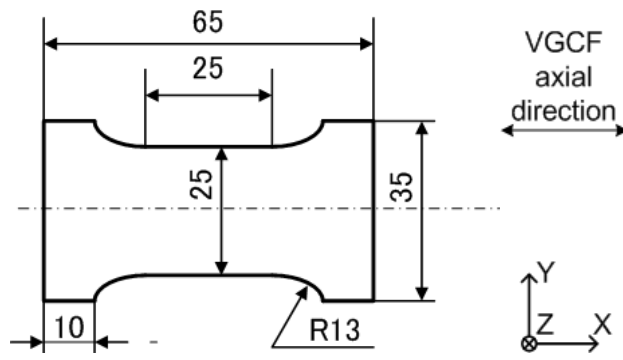

Fig. 6 Geometry of test specimens.(mm)

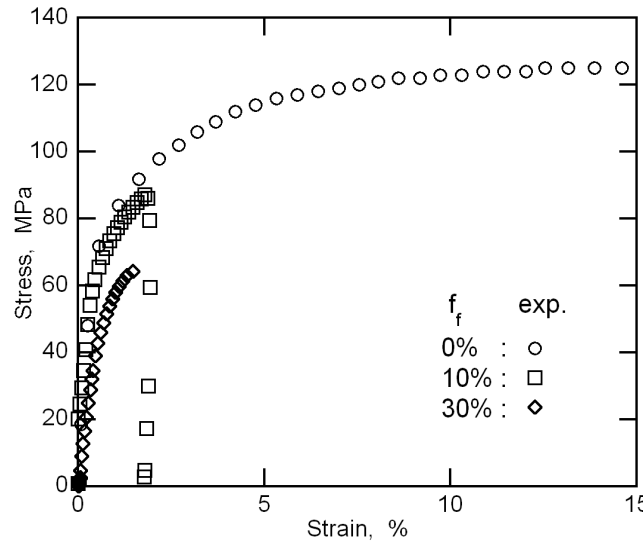

(a)still fracture[22]

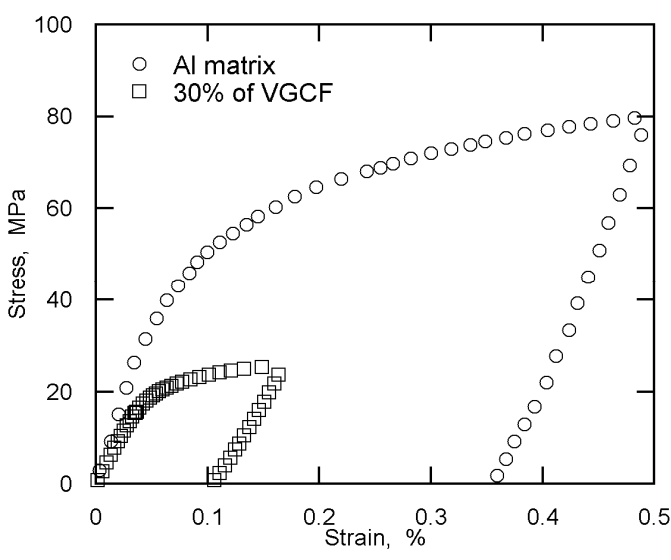

(b)prestrain

Fig. 7 Example of stress-strain relations of the composites.

Table 1 Strength properties of each volume fraction of VGCF.

\begin{tabular}{c|cc}
\hline $\begin{array}{c}\text { Volume fraction } \\
\text { of VGCF, \% }\end{array}$ & Tensile strength, MPa & Elongation, \% \\
\hline 0 & $125 \sim$ & $15 \sim$ \\
10 & 86 & 1.9 \\
30 & 64 & 1.4 \\
\hline
\end{tabular}

The tensile tests are conducted at room temperature with a crosshead speed of $0.3 \mathrm{~mm} / \mathrm{min}$. After the tensile loading, the test specimens are unloaded with the same crosshead speed. The tensile direction is the axial direction of the VGCF, and the testing apparatus is Ez Graph 5kN (SHIMADZU Co.), stresses are measured by a load cell and strains by a strain gauge (KYOWA Electronic Instrument Co., KFG-1-120-C1-16). After the tensile deformation, test specimens for measuring the thermal conductivity $(25 \mathrm{~mm}$ square and thickness of $3 \mathrm{~mm}$ ) are cut out from the tensile test specimens. The thermal conductivity in the $\mathrm{Z}$ direction is determined using the laser flash method and the value in the $\mathrm{X}$ and $\mathrm{Y}$ directions are determined using the two dimensional laser flash method[25 27]. This method can estimate the thermal conductivity in the $\mathrm{X}$ and $\mathrm{Y}$ directions of composites with satisfactory accuracy.

\subsection{Experimental results}

Figure 7 shows examples of the stress-strain relations of the composites. Figure 7(a) showed stress-strain relations still fracture[22]. Figure 7(b) shows stress-strain relation and the circles and squares show the stress-strain relations of the matrix and the composite containing $30 \%$ of VGCF, respectively. The matrix specimen is also fabricated using SPS method as same as the specimen of the composite. According to Fig.7(a), table 1 showed the strength properties of the composites. The tensile strength and elongation of Al matrix is over $125 \mathrm{MPa}$ and $15 \%$, respectively. The composites tended to be brittle and the tensile 


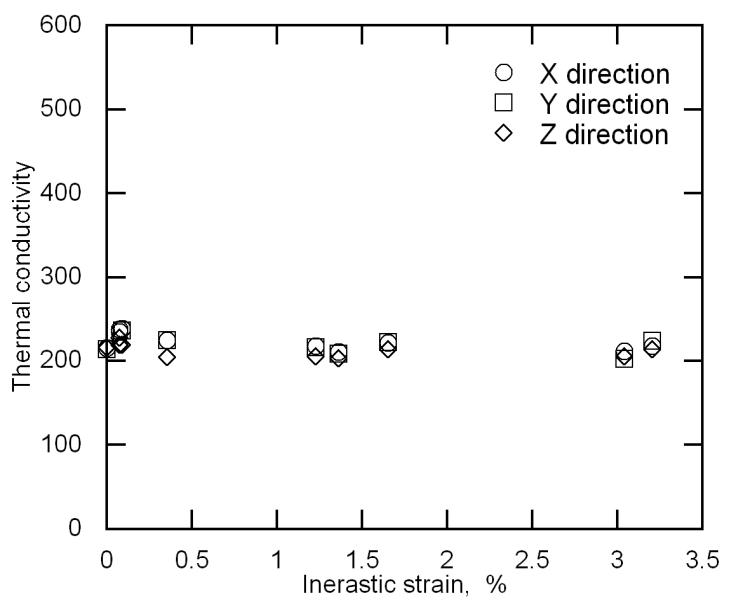

Fig. 8 Relationship between thermal conductivity and inelastic strain (matrix).

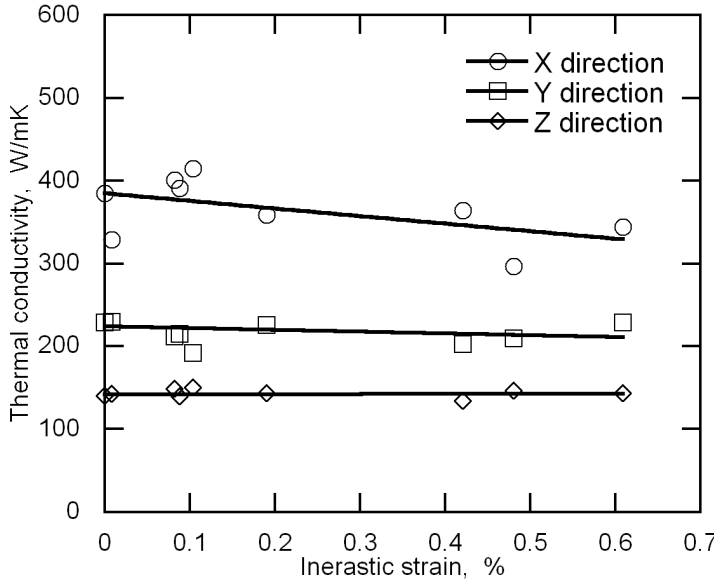

Fig. 9 Relationship between thermal conductivity and inelastic strain (30\% of VGCF).

strength of the composite decreased by the addition of VGCF as also reported elsewhere [20, 22].

Figure 8 shows the relationship between the thermal conductivity of the matrix and the inelastic strain due to the tensile loading. In Fig. 8, circles show the thermal conductivity of the $\mathrm{X}$ direction, squares that of the $\mathrm{Y}$ direction, and diamonds are for the $\mathrm{Z}$ direction. The thermal conductivity of the matrix does not change due to the inelastic deformation, i.e. thermal conductivity of the matrix is not affected by the inelastic deformation.

Figure 9 shows the relationship between the thermal conductivity and the inelastic strain for the composite containing a 30\% volume fraction of VGCF. The solid lines in Fig. 9 are the results of the least squares method. The correlation coefficients for the dependence of thermal conductivities on inelastic strain are calculated. The value of $\mathrm{X}, \mathrm{Y}$, and $\mathrm{Z}$ directions are $-0.54,-0.07$, and -0.13 , respectively. According to the results, the thermal conductivities in the $\mathrm{X}$ direction decrease with increases in the inelastic strain, while the thermal conductivities in the $\mathrm{Y}$ and $\mathrm{Z}$ directions do not change due to the inelastic deformation. It is hypothesized that the decrease in thermal conductivity of the $\mathrm{X}$ direction due to the inelastic strain comes from changes in microstructures. Therefore FEM analysis is conducted to explain the effect of the inelastic deformation on the thermal conductivities.

\section{Analyses}

\subsection{Thermal analysis using VGCF-CNT network models}

To explain the relationship between the thermal conductivity and the inelastic 


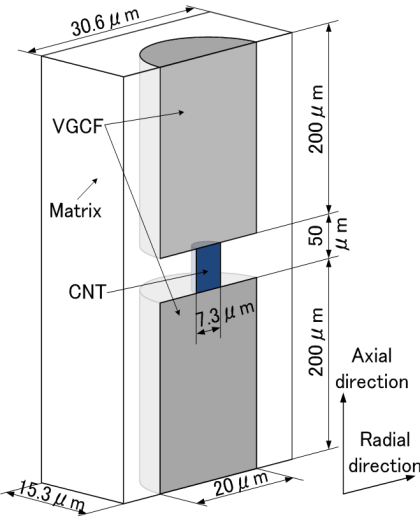

(a) geometry of the network model (N-D model)

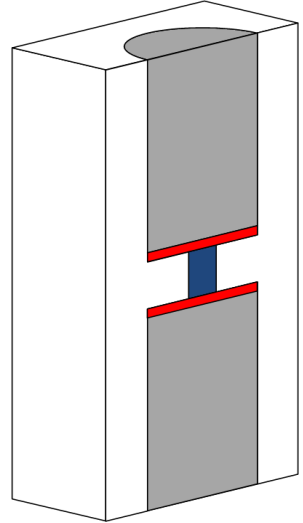

(c) axial debonding (A-D model)

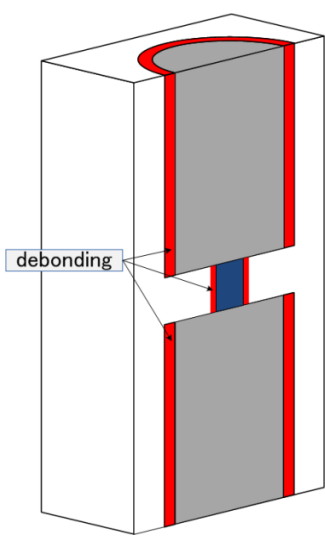

(b) radial debonding (R-D model)

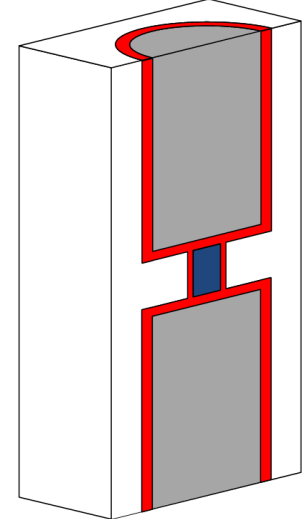

(d) both debonding (B-D model)

Fig. 10 Geometries of the analysis model considering the VGCF-CNT network.

Table 2 Thermal conductivity of materials. [5, 28]

\begin{tabular}{c|c}
\hline Material & Thermal conductivity $(\mathrm{W} / \mathrm{mK})$ \\
\hline Aluminum & 230 \\
VGCF axial direction & 2000 \\
VGCF radial direction & 10 \\
CNT axial direction & 6000 \\
CNT radial direction & 10 \\
Debonding & $1 \times 10^{-5}$ \\
\hline
\end{tabular}

deformation, a FEM analysis was conducted using ANSYS, software.

Figure 10 shows FEM models of the VGCF-CNT network assumed referring to the SEM micrograph in Fig. 5(a). Four FEM models were employed: a VGCF-CNT network without damage such as fiber debonding (Fig. 10(a), N-D model), a network with debonding in the radial direction (Fig. 10(b), R-D model), a network with debonding in the axial direction (Fig. 10(c), A-D model), and a network with debonding in both the radial and axial directions (Fig. 10(d), B-D model). In the case that the specimen was subjected to only the tensile loading along to the axial direction of VGCF, the debonding of the radial direction of VGCF occurred referring [22]. Therefore the R-D model in Fig. 10(b) and B-D model in Fig. 10(d) were considered. The size of VGCF elements is $20 \mu \mathrm{m}$ in diameter and $400 \mu \mathrm{m}$ in length, while the length of CNT is $50 \mu \mathrm{m}$. The diameter of the CNT and the width of the matrix are determined by considering the volume fraction of VGCF and CNT. The thickness of the debonding in the radial direction is $2 \%$ of the diameter of the fibers, while that of the debonding in the axial direction is $2 \%$ of the diameter of the VGCF. The volume fractions of VGCF and CNT are $30 \%$ and $0.5 \%$, respectively.

Table 2 shows the thermal conductivity of each material used in the analysis. Both the 


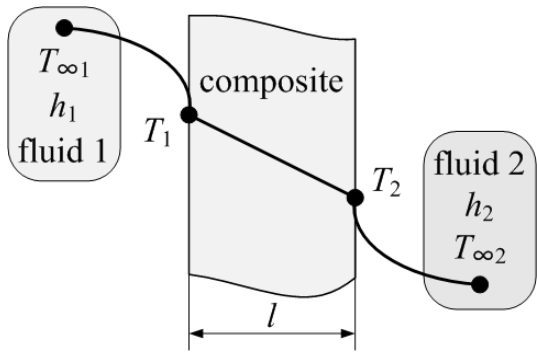

Fig. 11 Schematic figure of boundary condition.

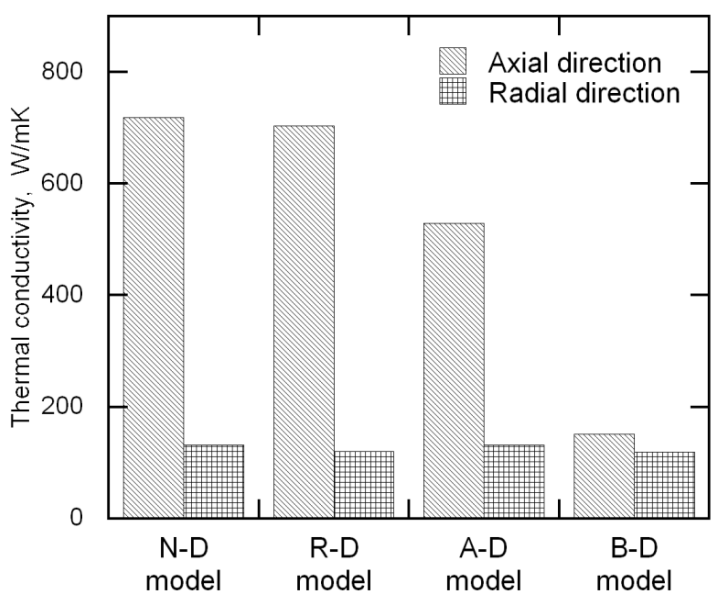

Fig. 12 Effect of the debonding on the thermal conductivity of the composites, simulated.

matrix is defined as thermal isotropy material, while VGCF and CNT are thermal anisotropy materials, respectively $[5,28]$. The debonding is assumed to be air close to the vacuum, which has quite low thermal conductivity.

Figure 11 shows schematic figure of boundary condition. Steady state heat conduction through the plane wall is assumed. According to one dimensional heat conduction equation,the thermal conductivity of the composites $\lambda_{\text {comp }}[\mathrm{W} / \mathrm{mK}]$ is expressed by the following Eq. (1).

$$
\lambda_{\text {comp }}=l \frac{h_{1} h_{2}}{h_{1}+h_{2}}\left(\frac{T_{\infty 1}-T_{\infty 2}}{T_{1}-T_{2}}-1\right)
$$

where $l$ is the thickness of the plane wall; $h_{1}$ and $h_{2}$ are the heat transfer coefficients of the fluid at high and low temperatures; $T_{\infty 1}$ and $T_{\infty 2}$ are the high and low temperatures of the fluid; $T_{1}$ and $T_{2}$ are the temperatures of wall surfaces in contact with the fluid at high and low temperatures. The values of these parameters are as follows: $h_{1}$ and $h_{2}$ are $10\left[\mathrm{~W} / \mathrm{m}^{2} \mathrm{~K}\right]$; $T_{\infty 1}$ and $T_{\infty 2}$ are $393 \mathrm{~K}$ and $293 \mathrm{~K} ; T_{1}$ and $T_{2}$ are obtained by the FEM, finally leading to $\lambda_{\text {comp. }}$.

Figure 12 shows the thermal conductivities of the axial and radial directions simulated using the FEM models in Fig. 10. The debondings lead to decreases in the thermal conductivity in the axial direction. The extent of the decrease is different for different types of debonding, compared with the model without debonding ( $\mathrm{N}-\mathrm{D}$ model): the radial debonding (R-D model) gives rise to the smallest decrease, the decrease with the axial debonding (A-D model) is the second smallest, and the debonding in both directions (B-D model) leads to the largest decrease in the thermal conductivity.

For the radial direction as shown in Fig. 12, the effect of the debonding on the thermal conductivity in the radial direction is much smaller than that in the axial direction. There are only very small differences in the extent of the decrease due to the various types of 
Table 3 Rate of decrease in thermal conductivity in X direction using the network models.

\begin{tabular}{c|c}
\hline Type of debonding & Decrease in thermal conductivity (\%) \\
\hline Experiment & 9 \\
R-D model (Simulation) & 2 \\
A-D model (Simulation) & 26 \\
B-D model (Simulation) & 79 \\
\hline
\end{tabular}

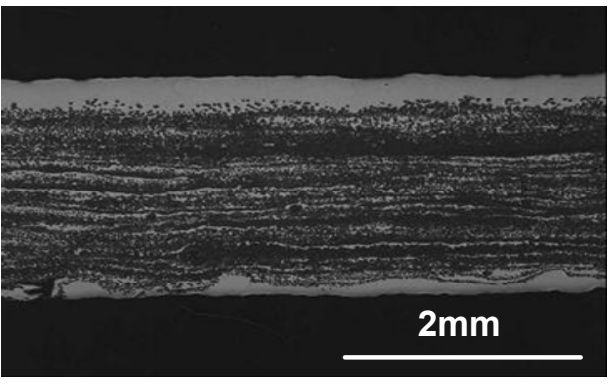

(a) low magnification

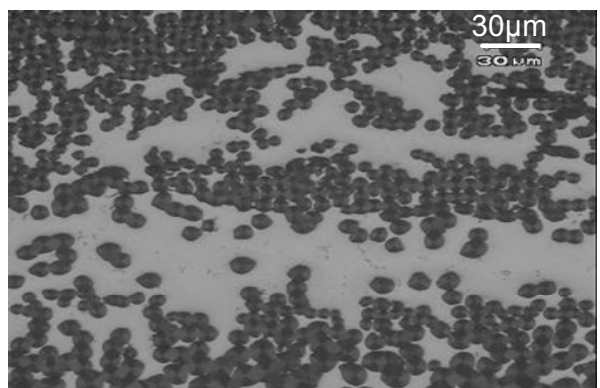

(b) high magnification

Fig. 13 SEM images of the composite (Y-Z plane)[22].

debonding. These results of both the axial and radial directions provide a qualitative substantiation of the experimental results.

These simulated thermal conductivities in the $\mathrm{X}$ direction are much larger than the measured values in Fig. 9: the thermal conductivity of the composite containing $30 \%$ of VGCF obtained by the experiments is $380 \mathrm{~W} / \mathrm{mK}$ while $718 \mathrm{~W} / \mathrm{mK}$ by the FEM analysis. This difference is caused as the analytical model in Fig. 10(a) does not consider porosity and agglomeration of the VGCF and CNT, which affect the thermal conductivity negatively. Therefore, the experiments and the simulation only allow a qualitative comparison of the results.

Table 3 shows the rate of decrease in thermal conductivity in the $\mathrm{X}$ direction of the composites. There is a large difference in the rate of decrease in thermal conductivity between the experiments and the simulations: the value of the rate of decrease obtained by the experiments was $9 \%$, while the simulation using the A-D model decreased $26 \%$. The network A-D model shown in Fig. 10(c) means that every VGCF-CNT networks in the composite has axial debonding due to inelastic deformation. In the actual materials, axial debonding occurs in parts of the VGCF-CNT network due to inelastic deformation. Comparing the experiments and the simulation, it can be estimated that $34 \%$ ( $9 \%$ vs $26 \%$ ) of the VGCF-CNT network consist of axial debonding.

\subsection{Thermal analysis considered macroscopic observation}

The fabricated composites in this study are regarded as laminate composites because the composites were fabricated by layering VGCF-CNT filler and matrix powder as detailed in section 2. Figure 13 showed SEM micrographs of a cross section area in the Y-Z plane of the composite in two magnifications[22]. It can be observed that the composite alternatively has matrix and filler layers.

Figure 14 shows FEM models referring to the SEM observations in Fig. 13. The FEM models in Fig. 14 have two filler layers and three matrix layers and some models have breaking part. The breaking models in Fig. 14 are considered assuming that the layer of the aligned VGCF containing CNT breaks due to the tensile deformation. The models have the length of $35 \mathrm{~mm}$, the width of $25 \mathrm{~mm}$ and the thickness of $3 \mathrm{~mm}$, respectively. Five FEM models are assumed: the filler layer does not have any breaking parts such as fiber breaking and debonding (Fig. 14(a), N-B model), the filler layer has a breaking part across in the Y direction (Fig. 14(b), A-B model), the filler layer has a breaking part from the side to the center (Fig. 14(c), H-B model), the filler layer has an internal breaking part from a quarter 


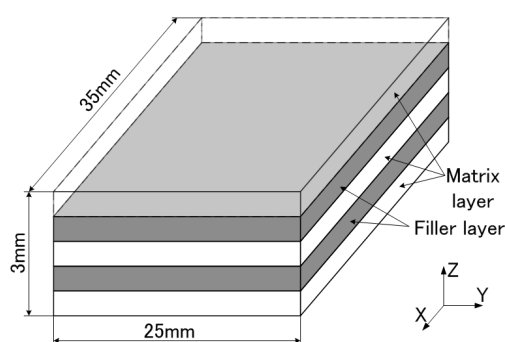

(a) geometry of the layer model (N-B model)

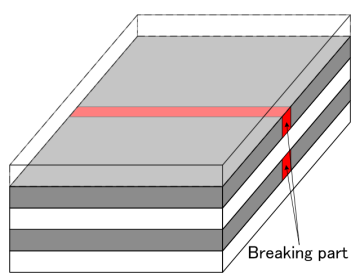

(b) all (A-B model)

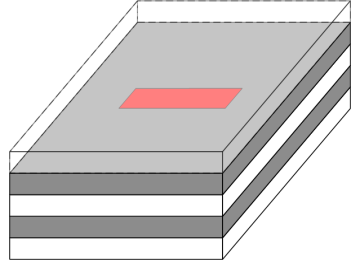

(d) center (C-B model)

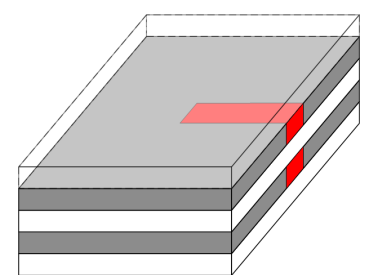

(c) half (H-B model)

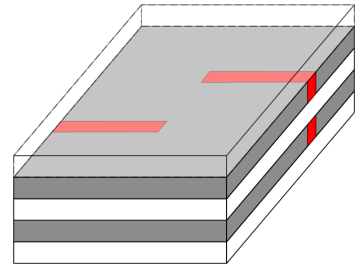

(e) two parts (T-B model)

Fig. 14 Geometries of the analysis model of the laminar composites.

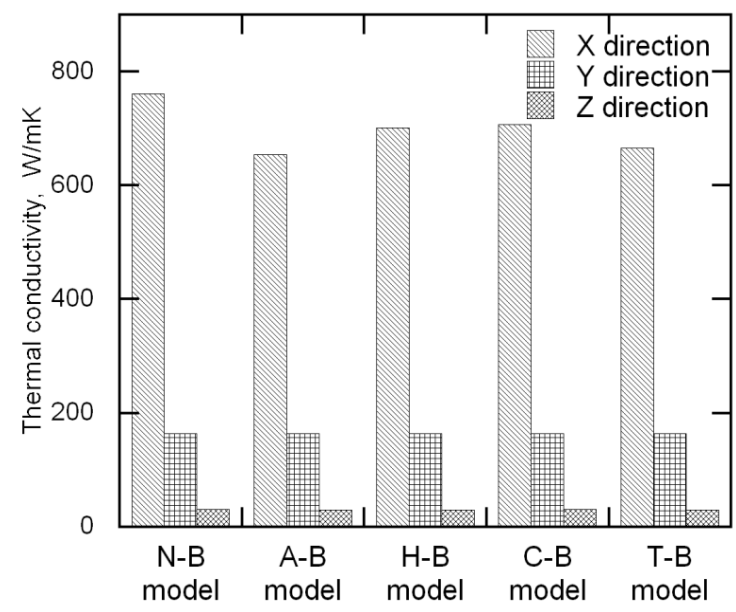

Fig. 15 Effect of breaking parts on the thermal conductivity of the composites, simulated.

to three quarters of the width (Fig. 14(d), C-B model), and the filler layer has two breaking parts from the side to the center (Fig. 14(e), T-B model). The thickness of the filler layer is determined by considering the volume fraction of VGCF, and the volume fraction of the breaking part is set as $1 \%$ of the volume fraction of VGCF. There is a possibility that the thermal conductivity changes due to the prestrain given the specimen. However a measurement of the size of the breaking part is quite difficult. In this paper, A-B model having the value of breaking part is $1 \%$ of VGCF is assumed following state; after tensile deformation, the inelastic deformation of the matrix layer, which is ductile material, is $1 \%$ for tensile direction, and the filler layer, which is brittle material, is broken due to tensile deformation.

The thermal conductivities of each material is used the value in Table 2. The X direction of the filler layer is the axial direction of VGCF and has the higher thermal conductivity $(2000 \mathrm{~W} / \mathrm{mK})$. The $\mathrm{Y}$ and $\mathrm{Z}$ directions of filler layer are same as the radial direction of VGCF $(10 \mathrm{~W} / \mathrm{mK})$. Matrix layer is same as Aluminum and the thermal conductivity is $230 \mathrm{~W} / \mathrm{mK}$. The thermal conductivity of the breaking part is equal to that of the debonding in Fig. $10\left(1 \times 10^{-5} \mathrm{~W} / \mathrm{mK}\right)$. The FEM analysis is conducted for the $30 \%$ volume fraction of VGCF.

Figure 15 shows the thermal conductivities simulated using each layer model. In the $\mathrm{Y}$ and $\mathrm{Z}$ direction, there are no differences in thermal conductivities for the different types of the breaking parts. However, in the $\mathrm{X}$ direction, there are differences in the thermal conductivity due to the types of breaking part: the A-B model has the smallest thermal 
Table 4 Rate of decrease in thermal conductivity in X direction using the layer models.

\begin{tabular}{c|c}
\hline Type of breaking part & Decrease in thermal conductivity (\%) \\
\hline Experiment & 9 \\
A-B model (Simulation) & 14 \\
H-B model (Simulation) & 8 \\
C-B model (Simulation) & 7 \\
T-B model (Simulation) & 12 \\
\hline
\end{tabular}

conductivity, because the breaking part across the $\mathrm{Y}$ direction completely blocks heat transfer in the $\mathrm{X}$ direction. The T-B model has the second smallest thermal conductivity in the $\mathrm{X}$ direction for the same reason.

Table 4 shows the rates of decrease in thermal conductivity in the $\mathrm{X}$ direction for the composite containing $30 \%$ of VGCF due to each breaking parts. The simulated decrease using the layer A-B (Fig. 14(b)) and H-B models (Fig. 14(c)) are 14\% and 8\%, respectively. The rate of decrease measured for the experiments is $9 \%$. It suggests that the layer H-B model provides the better estimate among the different layer models.

In this paper, the effect of the inelastic deformation on the thermal conductivity was observed using two types of idealized model. The simulation qualitatively shows the effect of the inelastic deformation on the thermal conductivity. However, to conduct more precise simulation using a real model, detailed observation of the microstructure should be needed. The observation are proceeded to make a real model.

\section{Conclusions}

This paper treated the effects of inelastic deformation on the thermal conductivity of VGCF/Al composites with dispersed CNT from both experiments and simulation. The thermal conductivities of the composites before and after tensile deformation were measured by experiments. A FEM analysis of the effect of inelastic deformation on the thermal conductivity of the composites was also conducted. The results suggested the following conclusions:

1) The thermal conductivity of the composites in the axial direction of the VGCF decreases with increases in inelastic deformation due to the tensile deformation. While that in the radial direction of the VGCF does not change before and after the inelastic deformation.

2) The effect of the inelastic deformation on thermal conductivity is not large: the thermal conductivity decreases about $9 \%$ due to the inelastic deformation. This means the composites fabricated here have thermal conductive properties processing a resistance to the inelastic deformation and the reliability of the composite is improved.

3) The effects of the inelastic deformation on the thermal conductivity in each direction can be qualitatively simulated using FEM analysis considering both the microscopic and macroscopic conditions.

4) Comparison of the simulations with experiments shows that there is a possibility to estimate changes in the internal structure of the composite due to the inelastic deformation.

\section{References}

(1) S. Iijima : Helical microtubules of graphitic carbon, Nature 354, 56-58(1991).

(2) C. Stephan, T. P. Nguyen, M. L. Chapelle, S. Lefrant, C. Journet, and P. Bernie : Characterization of Singlewalled Nanotubes-PMMA Composites, Synthetic Metals 108, 139-149 (2000).

(3) J. Hone, M. C. Llaguno, N. M. Nemes, A. T. Johnson, J. E. Fischer, D. A. Walters, M. J. Casavant, J. Schmidt, and R. E. Smalley : Electrical and Thermal Transport Properties of 
Magnetically Aligned Single Wall Carbon Nanotube Films, Applied Physics Letter, 77, 666-668(2000).

(4) J. Hone, M. C. Llaguno, M. J. Biercuk, A. T. Johnson, B. Batlogg, Z. Benes, and J. E. Fischer : Thermal Properties of Carbon Nanotubes and Nanotube-Based Materials, Applied Physics A Materials Science \& Processing, A74, 339-343 (2002).

(5) M. J. Biercuk, M. C. Llaguno, M. Radosavljevic, J. K. Hyun, A. T. Johnson, and J. E. Fischer : Carbon Nanotube Composites for Thermal Management, Applied Physics Letter, 80, 2767-2769(2002).

(6) R. D. Patton, C. U. Pittman Jr, L. Wang, J. R. Hill, A. Day : Ablation, mechanical and thermal conductivity properties of vapor grown carbon fiber/phenolic matrix composites. Composites Part A, 33, 243-251(2002).

(7) J. Chen, X. Xiong, and P. Xiao : The effect of MWNTs on the microstructure of resin carbon and thermal conductivity of C/C composites, Solid State Sciences, 11, 1890-1893(2009).

(8) T. H. Nam, S. Ogihara, N. H. Tung, S. Kobayashi : Mechanical and thermal properties of short coir fibre reinforced poly(butylene succinate) biodegradable composites, Journal of Solid Mechanics and Materials Engineering, 6, 251-262 (2011).

(9) J. Xu, Timothy S. Fisher : Enhancement of thermal interface materials with carbon nanotube arrays, International Journal of Heat and Mass Transfer, 49, 1658-1666 (2006).

(10) T. Wei, Z. Fan, G. Luo, and F. Wei : A new structure for multi-walled carbon nanotubes reinforced alumina nanocomposite with high strength and toughness, Materials Letters, 62, 641-644(2007).

(11) T. Tokunaga, K. Kaneko, Z. Horita : Production of aluminum-matrix carbon nanotube composite using high pressure torsion., Material Science and Engineering A, 490, 300-304, (2008).

(12) K. Ozawa, S. Song, and S. Yamauchi : Development of high thermal conductivity Al-Si/C/VGCF composites with C/VGCF form., TANSO 229, 233-236, (2007).

(13) Z. F. Xu, Y. B. Choi, K. Matsugi, D. C. Li, G. Sasaki : Mechanical and thermal properties of vapor-grown carbon fiber reinforced Aluminum matrix composites by plasma sintering, Materials transactions, 51, 510-515, (2010).

(14) T. Ueno, T. Yoshioka, J. Ogawa, K. Sato, K. Yoshino : Highly thermal conductive metal/ carbon composites by pulsed electric current sintering, Synthetic metals, 159, 2170-2172,(2009).

(15) I. V. Singh, M. Tanaka, M. Endo : Effect of interface on the thermal conductivity of carbon nanotube composites., International Journal of Thermal Science, 46, 842-827(2007).

(16) C-W. Nan, R. Birringer, D. R. Clarke, and H. Gleiter : Effective Thermal Conductivity of Particulate Composites with Interfacial Thermal Resistance, Journal of Applied Physics, 81, 6692-6699(1997).

(17) C-W. Nan, G. Liu., Y. Lin,and M. Li,:Interface Effect on Thermal Conductivity of Carbon Nanotube Composites, Applied Physics Letters, 85, 3549-3551(2004).

(18) J. M. Banet, Y. H. Zhao and G. J. Weng : Progressive debonding of aligned oblate inclusions and loss of stiffness in a brittle matrix composite, Eng. Frac. Mech., 53, 897-910(1996).

(19) S. Y. Chang, C. C. Jain, T. H. Chuang, L. P. Feng, L. C. Tsao : Effect of addition of TiO2 nanoparticle on the micriostructure, microhardness and interfacial reactions of Sn3.5AgXCu solder, Materials and Design, 32, 4720-4727, (2011).

(20) T. Imanishi, K. Sasaki, K. Katagiri, and A. Kakitsuji : Thermal and Mechanical Properties of VGCF-containing Aluminum, Transactions of the Japanese society of mechanical engineers, 74, 651-655(2008) (in Japanese).

(21) T. Imanishi, K. Sasaki, K. Katagiri, K. Katagiri, and A. Kakitsuji : Effect of CNT Addition on Thermal Properties of VGCF/Aluminum Composites, Transactions of the Japanese 
society of mechanical engineers, 75, 27-33(2009) (in Japanese).

(22) K. Fukuchi, K. Sasaki, T. Imanishi, K. Katagiri, A.Kakitsuji, A. Shimizu : Strength of VGCF/Al composites for high thermal conductivity, Journal of Solid Mechanics and Materials Engineering, 4, 1273-1281(2010).

(23) B. Lestriez, S. Desaever, J. Danet, P. Moreau, D. Plée, D. Guvomard : Hierarchical and Resilient Conductive Network of Bridged Carbon Nanotubes and Nanofibers for HighEnergy Si Negative Electrodes, Electrochemical and Solid-State Letters, 12, A76-A80 (2009).

(24) M. S. Wu, C.-Y. Huang, J.-J. Jow : Electrophoretic Deposition of Network-Like Carbon Nanofiber as a Conducting Substrate for Nanostructured Nickel Oxide Electrode, Electrochemistry Communications, 11, 779-782 (2009).

(25) T. Azumi : Two dimensional measuring technique of thermal diffusivity by flash method, Proc. 9th Jpn. Symp. Thermophys. Prop., A209, (1988)(in Japanese).

(26) T. Yamane, S. Katayama, and M. Todoki : Thermal diffusivity measurement of plate using flash method., Proc. 15th Jpn. Symp. Thermophys. Prop., C203, (1994)(in Japanese).

(27) Y. Agari : Measurement of effective thermal diffusivity of polymer composites in the film direction, Proc. 29th Jpn. Symp. Thermophys. Prop., A305, (2008)(in Japanese).

(28) J. Che, T. Cagin, W. A. Goddard : Thermal conductivity of carbon nanotubes, Nanotechnology, 11, 65-69 (2000). 